\title{
MAXIMAL SUBALGEBRAS AND CHIEF FACTORS OF LIE ALGEBRAS
}

\author{
DAVID A. TOWERS
}

\author{
Department of Mathematics and Statistics \\ Lancaster University \\ Lancaster LA1 4YF \\ England \\ d.towers@lancaster.ac.uk
}

\begin{abstract}
This paper is a continued investigation of the structure of Lie algebras in relation to their chief factors, using concepts that are analogous to corresponding ones in group theory. The first section investigates the structure of Lie algebras with a core-free maximal subalgebra. The results obtained are then used in section two to consider the relationship of two chief factors of $L$ being $L$-connected, a weaker equivalence relation on the set of chief factors than that of being isomorphic as $L$-modules. A strengthened form of the Jordan-Hölder Theorem in which Frattini chief factors correspond is also established for every Lie algebra. The final section introduces the concept of a crown, a notion introduced in group theory by Gaschütz, and shows that it gives much information about the chief factors

Mathematics Subject Classification 2010: 17B05, 17B20, 17B30, 17B50.

Key Words and Phrases: Lie algebras, maximal subalgebra, core-free, chief factor, crown, prefrattini subalgebras.
\end{abstract}




\section{Primitive algebras}

Throughout $L$ will denote a finite-dimensional Lie algebra over a field $F$. The symbol ' $\oplus$ ' will denote an algebra direct sum, whilst ' $\dot{+}$ ' will denote a direct sum of the underlying vector space structure alone. If $U$ is a subalgebra of $L$ we define $U_{L}$, the core (with respect to $L$ ) of $U$ to be the largest ideal of $L$ contained in $U$. We say that $U$ is core-free in $L$ if $U_{L}=0$. We shall call $L$ primitive if it has a core-free maximal subalgebra. The centraliser of $U$ in $L$ is $C_{L}(U)=\{x \in L:[x, U]=0\}$. Then we have the following characterisation of primitive Lie algebras.

Theorem 1.1 1. A Lie algebra $L$ is primitive if and only if there exists a subalgebra $M$ of $L$ such that $L=M+A$ for all minimal ideals $A$ of $L$.

2. Let $L$ be a primitive Lie algebra. Assume that $U$ is a core-free maximal subalgebra of $L$ and that $A$ is a non-trivial ideal of $L$. Write $C=$ $C_{L}(A)$. Then $C \cap U=0$. Moreover, either $C=0$ or $C$ is a minimal ideal of $L$.

3. If $L$ is a primitive Lie algebra and $U$ is a core-free maximal subalgebra of $L$, then exactly one of the following statements holds:

(a) $\operatorname{Soc}(L)=A$ is a self-centralising abelian minimal ideal of $L$ which is complemented by $U$; that is, $L=U \dot{+} A$.

(b) $\operatorname{Soc}(L)=A$ is a non-abelian minimal ideal of $L$ which is supplemented by $U$; that is, $L=U+A$. In this case $C_{L}(A)=0$.

(c) $\operatorname{Soc}(L)=A \oplus B$, where $A$ and $B$ are the two unique minimal ideals of $L$ and both are complemented by $U$; that is, $L=A \dot{+} U=$ $B \dot{+} U$. In this case $A=C_{L}(B), B=C_{L}(A)$, and $A, B$ and $(A+B) \cap U$ are non-abelian isomorphic Lie algebras.

\section{Proof.}

1. If $L$ is primitive and $U$ is a core-free maximal subalgebra then it is clear that $L=U+A$ for every minimal ideal $A$ of $L$. Conversely, if there exists a subalgebra $M$ of $L$ such that $L=M+A$ for every minimal ideal $A$ of $L$ and $U$ is a maximal subalgebra of $L$ such that $M \subseteq U$, then $U$ cannot contain any minimal ideal of $L$, and therefore $U$ is a core-free maximal subalgebra of $L$. 
2. Since $U$ is core-free in $L$, we have that $L=U+A$. Since $A$ is an ideal of $L$, then $C$ is an ideal of $L$ and then $C \cap U$ is an ideal of $U$. Since $[C \cap U, A]=0$, we have that $C \cap U$ is an ideal of $L$. Therefore $C \cap U=0$.

If $C \neq 0$, consider a minimal ideal $X$ of $L$ such that $X \subseteq C$. Since $X \nsubseteq U$, then $L=X+U$. But now $C=C \cap(X+U)=X+(C \cap U)=X$.

3. Let us assume that $A_{1}, A_{2}, A_{3}$ are three pairwise distinct minimal ideals of $L$. Since $A_{1} \cap A_{2}=A_{1} \cap A_{3}=A_{2} \cap A_{3}=0$, we have that $A_{2} \oplus A_{3} \subseteq C_{L}\left(A_{1}\right)$. But then $C_{L}\left(A_{1}\right)$ is not a minimal ideal of $L$, and this contradicts 2 . Hence, in a primitive Lie algebra there exist at most two distinct minimal ideals.

Suppose that $A$ is a non-trivial abelian ideal of $L$. Then $A \subseteq C_{L}(A)$. Since by $2, C_{L}(A)$ is a minimal ideal of $L$, we have that $A$ is selfcentralising. Thus, in a primitive Lie algebra there exists at most one abelian minimal ideal of $L$. Moreover, $L=A+U$ and $A$ is selfcentralising. Then $A \cap U=C_{L}(A) \cap U=0$.

If there exists a unique minimal non-abelian ideal $A$ of $L$, then $L=$ $A+U$ and $C_{L}(A)=0$.

If there exist two minimal ideals $A$ and $B$, then $A \cap B=0$ and then $B \subseteq C_{L}(A)$ and $A \subseteq C_{L}(B)$. Since $C_{L}(A)$ and $C_{L}(B)$ are minimal ideals of $L$, we have that $B=C_{L}(A)$ and $A=C_{L}(B)$. Now $A \cap U=$ $C_{L}(B) \cap U=0$ and $B \cap U=C_{L}(A) \cap U=0$. Hence $L=A \dot{+} U=B \dot{+} U$. Since $A=C_{L}(B)$, it follows that $B$ is non-abelian. Analogously we have that $A$ is non-abelian. Furthermore, we have $A+((A+B) \cap U)=$ $A+B=B+((A+B) \cap U)$. Hence

$$
A \cong \frac{A}{A \cap B} \cong \frac{A+B}{B} \cong \frac{B+((A+B) \cap U)}{B} \cong(A+B) \cap U .
$$

Analogously $B \cong(A+B) \cap U$.

As in the group-theoretic case this leads to three types. A primitive Lie algebra is said to be

1. primitive of type 1 if it has a unique minimal ideal that is abelian;

2. primitive of type 2 if it has a unique minimal ideal that is non-abelian; and 
3. primitive of type 3 if it has precisely two distinct minimal ideals each of which is non-abelian.

Of course, primitive Lie algebras of types 2 and 3 are semisimple, and those of types 1 and 2 are monolithic. (A Lie algebra $L$ is called monolithic if it has a unique minimal ideal $W$, the monolith of $L$.) Examples of each type are easy to find.

EXAMPLE 1.1 1. Clearly every primitive solvable Lie algebra is of type 1.

2. Every simple Lie algebra is primitive of type 2 .

3. If $S$ is a simple Lie algebra then $L=S \oplus S$ is primitive of type 3 with core-free maximal subalgebra $D=\{s+s: s \in S\}$, the diagonal subalgebra of $L$.

Let $M$ be a maximal subalgebra of $L$. Then $M / M_{L}$ is a core-free maximal subalgebra of $L / M_{L}$. We say that $M$ is

1. a maximal subalgebra of type 1 if $L / M_{L}$ is primitive of type 1 ;

2. a maximal subalgebra of type 2 if $L / M_{L}$ is primitive of type 2 ; and

3. a maximal subalgebra of type 3 if $L / M_{L}$ is primitive of type 3 .

Lemma 1.2 Let $L$ be a non-trivial Lie algebra.

(i) If $M$ is a maximal subalgebra of $L$, then $L / M_{L}$ is primitive.

(ii) If $B$ is an ideal of $L$ and $L / B$ is primitive, then $L$ has a maximal subalgebra $M$ such that $B=M_{L}$.

\section{Proof.}

(i) This is easy.

(ii) Let $M / B$ be a core-free maximal subalgebra of $L / B$. Then $M$ is a maximal subalgebra of $L$ and $M_{L}=B$.

We say that an ideal $A$ is complemented in $L$ if there is a subalgebra $U$ of $L$ such that $L=A+U$ and $A \cap U=0$. For primitive solvable Lie algebras we have the following analogue of Galois' Theorem for groups. 
Theorem 1.3 1. If $L$ is a solvable primitive Lie algebra then all core-free maximal subalgebras are conjugate.

2. If $A$ is a self-centralising minimal ideal of a solvable Lie algebra L, then $L$ is primitive, $A$ is complemented in $L$, and all complements are conjugate.

\section{Proof.}

1. This is [8, Lemma 3].

2. This follows easily from [6, Lemma 1.5] and the part 1.

The Frattini ideal of $L, \phi(L)$, is the core of intersection of the maximal subalgebras of $L$. We say that $L$ is $\phi$-free if $\phi(L)=0$. Then we have the following characterisation of primitive Lie algebras of type 1.

Theorem 1.4 Let $L$ be a Lie algebra over a field $F$.

1. $L$ is primitive of type 1 if and only if $L$ is monolithic, with abelian monolith $W$, and $\phi$-free.

2. If $F$ has characteristic zero, then $L$ is primitive of type 1 if and only if $L=W \ltimes(C \oplus S)$, where $W$ is the abelian monolith of $L, C$ is an abelian subalgebra of $L$, every element of which acts semisimply on $W$, and $S$ is a Levi subalgebra of $L$.

3. If $L$ is solvable, then $L$ is primitive if and only if it has a self-centralising minimal ideal $A$.

\section{Proof.}

1. If $L$ is primitive of type 1 then it has the stated properties, by Theorem 1.1. So suppose that $L$ is monolithic with abelian monolith $W$ and $\phi$ free. Then there is a maximal subalgebra $M$ of $L$ such that $L=W \dot{+} M$. If $M_{L} \neq 0$ there is a minimal ideal of $L$ contained in $M_{L}$ and distinct from $W$, a contradiction. Hence $M_{L}=0$ and $L$ is primitive of type 1 .

2. This follows from 1 and [5, Theorem 7.5].

3. If $L$ is primitive then it has the stated property, by Theorem 1.1. The converse follows from [6, Lemma 1.5]. 
Theorem 1.5 For a Lie algebra $L$ the following are pairwise equivalent:

1. L is primitive of type 1 or 3 ;

2. there is a minimal ideal $B$ of $L$ complemented by a subalgebra $U$ which also complements $C_{L}(B)$;

3. there is a minimal ideal $B$ of $L$ such that $L$ is isomorphic to the semidirect sum $X=B \ltimes L / C_{L}(B)$.

\section{Proof.}

$1 \Rightarrow 2$ : This is clear from Theorem 1.1.

$2 \Rightarrow 1$ : Since $U_{L} \cap B=0$ we have $U_{L} \subseteq C_{L}(B)$. But now $U_{L} \cap C_{L}(B)=0$ implies that $U_{L}=0$. Suppose that $M$ is a proper subalgebra of $L$ containing $U$. Then $M \cap B$ is an ideal of $M$ and is centralised by $C_{L}(B)$, so $M \cap B$ is an ideal of $M+C_{L}(B)=L$. By the minimality of $B$ we have that $M \cap B=0$ and $U=M$. It follows that $U$ is a core-free maximal subalgebra of $L$ and $L$ is primitive. Finally note that the minimal ideal of a primitive Lie algebra of type 2 has trivial centraliser.

$2 \Rightarrow 3$ : Simply note that $L=B \dot{+} U$ and $U \cong L / C_{L}(B)$, whence the map $\theta: B \ltimes L / C_{L}(B) \rightarrow L$ defined by $\theta(b+u)=b+\left(u+C_{L}(B)\right)$ is the required isomorphism.

$3 \Rightarrow 2:$ Put $C=C_{L}(B)$ and assume there is an isomorphism $\theta: L \rightarrow$ $B \ltimes L / C$. Consider the following subalgebras: $B^{*}=\theta(\{b+C: b \in B\})$, $U^{*}=\theta\left(\{(0+(x+C): x \in L\})\right.$ and $C^{*}=\theta(\{b+(x+C): b+x \in C\})$. For each $b \in B$, we have that $\theta(-b+(b+C))$ is a non-trivial element of $C^{*}$, and so $C^{*} \neq 0$. It is easy to check that $B^{*}$ is a minimal ideal of $L$, that $C^{*}=C_{L}\left(B^{*}\right)$ and that $U^{*}$ complements $B^{*}$ and $C^{*}$.

As usual, $\mathcal{O}(m ; \underline{1})$ denotes the truncated polynomial ring in $n$ indeterminates defined over a field of characteristic $p>0$. Then the above yields the following characterisation of primitive Lie algebras of type 3 .

Corollary 1.6 1. L is primitive of type 3 if and only if $L$ has two distinct minimal ideals $B_{1}$ and $B_{2}$ with a common complement and such that the factor algebras $L / B_{i}$ are primitive of type 2 for $i=1,2$. 
2. If $F$ is algebraically closed, then, in addition to $1, B_{1}$ and $B_{2}$ are both isomorphic to $S \otimes \mathcal{O}(m ; \underline{1})$, where $S$ is simple. In this case

$$
L \subseteq \bigoplus_{i=1}^{2}\left(\operatorname{Der} S_{i} \otimes \mathcal{O}(m ; \underline{1})\right) \oplus\left(I_{S_{i}} \otimes W(m ; \underline{1})\right),
$$

where $S_{1} \cong S_{2} \cong S$.

3. If $F$ has characteristic zero, then $L$ is primitive of type 3 if and only if $L=S \oplus S$, where $S$ is simple.

\section{Proof.}

1. Suppose first that $L$ is primitive of type 3. Then $L$ has two distinct minimal ideals $B_{1}$ and $B_{2}$ which have a common complement $U$ in $L$, by Theorem 1.1. Also, $U \cong L / B_{1}$ and $\left(B_{2}+B_{1}\right) / B_{1}$ is a minimal ideal of $L / B_{1}$. If $x+B_{1} \in C_{L / B_{1}}\left(\left(B_{2}+B_{1}\right) / B_{1}\right)$ then $[b, x] \in B_{1}$ for all $b \in B_{2}$, which yields that $[x, b] \in B_{1} \cap B_{2}=0$ and so $x \in C_{L}\left(B_{2}\right)=B_{1}$. Hence $C_{L / B_{1}}\left(\left(B_{2}+B_{1}\right) / B_{1}\right)=0$, implying that $L / B_{1}$ is primitive of type 2 , and therefore so are $U$ and $L / B_{2}$.

Conversely, suppose that $L$ has two distinct minimal ideals $B_{1}$ and $B_{2}$ with a common complement $U$ and such that the factor algebras $L / B_{i}$ are primitive of type 2 for $i=1,2$. Then $U \cong L / B_{i}$ is primitive of type 2 such that $\operatorname{Soc}\left(L / B_{i}\right)=\left(B_{1}+B_{2}\right) / B_{i}$ and $C_{L}\left(\left(B_{1}+B_{2}\right) / B_{i}\right)=B_{i}$. It follows that $C_{L}\left(B_{2}\right)=B_{1}$ and $C_{L}\left(B_{1}\right)=B_{2}$. By Theorem 1.5 this implies that $L$ is primitive of type 3 .

2. This follows from Block's Theorem in [1].

3. If $L$ is primitive of type 3 it must be semisimple with precisely two ideals which are isomorphic to each other, and thus is as described.

This leaves primitive Lie algebras of type 2 , which can be characterised as follows.

Theorem 1.7 1. If $F$ is algebraically closed, then $L$ is primitive of type 2 if and only if

$$
L \cong U+(S \otimes \mathcal{O}(m ; \underline{1})) \subseteq(\operatorname{Der} S \otimes \mathcal{O}(m ; \underline{1})) \oplus\left(I d_{S} \otimes W(m ; \underline{1})\right),
$$

where $S \otimes \mathcal{O}(m ; \underline{1})$ is an ideal of $L$ and $S$ is simple. 
2. If $F$ has characteristic zero, then $L$ is primitive of type 2 if and only if $L$ is simple.

3. $L$ is primitive of type 2 if and only if there is a primitive Lie algebra $X$ of type 3 such that $L \cong X / B$ for a minimal ideal $B$ of $L$.

\section{Proof.}

1 and 2 are clear from Theorem 1.1.

3. Suppose that $L$ is primitive of type 2 , and let $D$ be the unique minimal ideal of $L$. Then $D$ is non-abelian and $C_{L}(D)=0$, so the semi-direct sum $X=D \ltimes L$ is primitive of type 3 , by Theorem 1.5. Clearly, if $B=\{b+0: b \in D\}$, then $X / B \cong L$. The converse follows easily from Corollary 1.6 and Theorem 1.1.

A special case of the above occurs as follows. We will call a Lie algebra $L$ almost simple if it is a subalgebra of Der $S$ for some simple subalgebra $S$ of $L$. Over a field of characteristic zero such an algebra has to be simple, as $L$ would be sandwiched between $\operatorname{Inn} S(\cong S)$ and Der $S$, and simple Lie algebras over such fields have no outer derivations. However, that is not the case for a field of positive characteristic $p$, even if it is algebraically closed and $S$ is restricted (see [3]). It is straightforward to check that $C_{\operatorname{Der}(S)}(S)=0$ and thus that an almost simple Lie algebra is primitive of type 2 .

\section{Chief factors}

We say that two chief factors are $L$-isomorphic, denoted by ' $\cong_{L}$ ', if they are isomorphic as $L$-modules. The centraliser of a chief factor $A / B$ is $C_{L}(A / B)=\{x \in L:[x, A] \subseteq B\}$.

Theorem 2.1 Let $L$ be a Lie algebra and let $A_{1} / B_{1}, A_{2} / B_{2}$ be two chief factors of $L$. Then

(i) if $A_{1} / B_{1}$ and $A_{2} / B_{2}$ are L-isomorphic, $C_{L}\left(A_{1} / B_{1}\right)=C_{L}\left(A_{2} / B_{2}\right)$;

(ii) if $A_{1} / B_{1}$ and $A_{2} / B_{2}$ are non-abelian, they are L-isomorphic if and only if $C_{L}\left(A_{1} / B_{1}\right)=C_{L}\left(A_{2} / B_{2}\right)$. 
Proof. (i) is clear, so suppose that $C=C_{L}\left(A_{1} / B_{1}\right)=C_{L}\left(A_{2} / B_{2}\right)$. Then $B_{i} \subseteq C \cap A_{i}$ for $i=1,2$. Since $A_{i} / B_{i}$ is non-abelian we have $A_{i} \not \subseteq C$, which yields that $B_{i}=C \cap A_{i}$ and $A_{i} / B_{i}$ is $L$-isomorphic to $\left(A_{i}+C\right) / C$ for $i=1,2$. Now $\left(A_{1}+C\right) / C$ is a minimal ideal of $L / C$ and $C_{L / C}\left(\left(A_{1}+C\right) / C\right)=C$, so $L / C$ is primitive of type 2 . But $\left(A_{2}+C\right) / C$ is also a minimal ideal of $L / C$, so we have that $A_{1}+C=A_{2}+C$. It follows that $A_{1} / B_{1}$ and $A_{2} / B_{2}$ are $L$-isomorphic.

It is easy see that 2.1(ii) does not hold for abelian chief factors, as the following example shows.

EXAMPLE 2.1 Let $L=\mathbb{R} a+\mathbb{R} b+\mathbb{R} c+\mathbb{R} x$ with multiplication $[x, a]=a$, $[x, b]=c,[x, c]=-b$. Then $A_{1}=\mathbb{R} a$ and $A_{2}=\mathbb{R} b+\mathbb{R} c$ are minimal ideal of $L$ and $C_{L}\left(A_{1}\right)=\mathbb{R} a+\mathbb{R} b+\mathbb{R} c=C_{L}\left(A_{2}\right)$, but $A_{1}$ and $A_{2}$ are clearly not L-isomorphic.

Recall that the Frattini subalgebra, $F(L)$, of $L$ is the intersection of the maximal proper subalgebras of $L$; the Frattini ideal, $\phi(L)$, is the largest ideal of $L$ contained in $F(L)$. Let $A / B$ be a chief factor of $L$. We say that $A / B$ is a Frattini chief factor if $A / B \subseteq \phi(L / B)$. In [7] a strengthened form of the Jordan-Hölder Theorem in which Frattini chief factors correspond was established for solvable Lie algebras. However, the assumption of solvability is not needed as we shall show below. This assumption is used only to establish [7, Lemma 2.1], so we simply need a slightly modified version of that Lemma.

Lemma 2.2 Let $A_{1}, A_{2}$ be distinct minimal ideals of the Lie algebra $L$. Then there is a bijection

$$
\theta:\left\{A_{1},\left(A_{1}+A_{2}\right) / A_{1}\right\} \rightarrow\left\{A_{2},\left(A_{1}+A_{2}\right) / A_{2}\right\}
$$

such that corresponding chief factors are isomorphic as L-modules and Frattini chief factors correspond to one another.

Proof. Put $A=A_{1} \oplus A_{2}$. Suppose first that $A_{1}$ is a Frattini chief factor. Then $A_{1} \subseteq \phi(L)$. Thus $A / A_{2} \subseteq \phi\left(L / A_{2}\right)$ and $A / A_{2}$ is a Frattini chief factor. If $A / A_{1}$ is also a Frattini chief factor, then $A / A_{1} \subseteq \phi\left(L / A_{1}\right)$, which yields that $A \subseteq \phi(L)$, and all four factors are Frattini. In this case we can choose $\theta$ so that $\theta\left(A_{1}\right)=A / A_{2}$ and $\theta\left(A / A_{1}\right)=A_{2}$. If $A / A_{1}$ is not a Frattini chief factor, then nor is $A_{2}$, by the same argument as above, and so the same choice of $\theta$ suffices; likewise if none of the factors are Frattini chief factors. 
The remaining case is where $A_{1}$ and $A_{2}$ are not Frattini chief factors but $A / A_{2}$ is. The fact that $A / A_{2}$ is a Frattini chief factor means that every maximal subalgebra containing $A_{2}$ also contains $A$, and so contains $A_{1}$. But, since $A_{1}$ is not a Frattini chief factor, there is a maximal subalgebra $M$ not containing $A_{1}$. It follows that $A_{2} \nsubseteq M$. Thus $L=M+A_{1}=$ $M+A_{2}$. Also, $\left[L, M \cap A_{1}\right]=\left[M+A_{2}, M \cap A_{1}\right] \subseteq M \cap A_{1}$. It follows that $M \cap A_{1}=0$. Similarly $M \cap A_{2}=0$. Put $C=A \cap M$. Then $L / A_{i} \cong M$ and this isomorphism maps the set of of maximal subalgebras of $L / A_{i}$ onto the set of maximal subalgebras of $M$. Since $A / A_{2}$ is a Frattini chief factor, every maximal subalgebra of $L$ containing $A_{2}$ contains $A$, so every maximal subalgebra of $M$ contains $C$. It follows that every maximal subalgebra of $L$ which contains $A_{1}$ also contains $C+A_{1}$; that is, $A / A_{1}$ is a Frattini chief factor of $L$. So we can choose $\theta$ so that $\theta\left(A_{1}\right)=A_{2}$ and $\theta\left(A / A_{1}\right)=A / A_{2}$.

Then the following result follows exactly as does [7, Theorem 2.2].

Theorem 2.3 Let

$$
\begin{aligned}
& 0<A_{1}<\ldots<A_{n}=L \\
& 0<B_{1}<\ldots<B_{n}=L
\end{aligned}
$$

be chief series for the Lie algebra L. Then there is a bijection between the chief factors of these two series such that corresponding factors are isomorphic as L-modules and such that the Frattini chief factors in the two series correspond.

Let $A / B$ be a chief factor of $L$. If there is a subalgebra $M$ such that $L=A+M$ and $B \subseteq A \cap M$, we say that $A / B$ is a supplemented chief factor of $L$, and that $M$ is a supplement of $A / B$ in $L$. If $A / B$ is a non-Frattini chief factor of $L$ then $A / B$ is supplemented by a maximal subalgebra $M$ of $L$.

If $A / B$ is a chief factor of $L$ supplemented by a subalgebra $M$ of $L$ and $A \cap M=B$ then we say that $A / B$ is a complemented chief factor of $L$, and $M$ is a complement of $A / B$ in $L$. When $L$ is solvable it is easy to see that a chief factor is Frattini if and only if it is not complemented.

Note that if $L$ is a primitive Lie algebra of type 3, its two minimal ideals are not $L$-isomorphic, so we introduce the following concept. We say that two chief factors of $L$ are $L$-connected if either they are $L$-isomorphic, or there exists an epimorphic image $\bar{L}$ of $L$ which is primitive of type 3 and 
whose minimal ideals are $L$-isomorphic, respectively, to the given factors. (It is clear that, if two chief factors of $L$ are $L$-connected and are not $L$ isomorphic, then they are nonabelian and there is a single epimorphic image of $L$ which is primitive of type 3 and which connects them.)

Theorem 2.4 The relation 'is L-connected to' is an equivalence relation on the set of chief factors.

Proof. The relation is clearly reflexive and symmetric, so we simply have to establish transitivity. Suppose that $A_{i} / B_{i}$ are chief factors of $L$ for $i=1,2,3$, for which $A_{1} / B_{1}$ is $L$-connected to $A_{2} / B_{2}$ and $A_{2} / B_{2}$ is $L$-connected to $A_{3} / B_{3}$. If any two are $L$-isomorphic the result is clear. So suppose that there are primitive epimorphic images images $L / K$ (with minimal ideals $H / K$ and $J / K$ ) and $L / T$ (with minimal ideals $P / T$ and $Q / T$ ) such that

$$
A_{1} / B_{1} \cong_{L} H / K, \quad J / K \cong_{L} A_{2} / B_{2} \cong_{L} P / T, \quad A_{3} / B_{3} \cong_{L} Q / T .
$$

Then $H=C_{L}(J / K)=C_{L}(P / T)=Q$. Now $L / H \cong(L / K) /(H / K)$, which is primitive of type 2 by Corollary 1.6. But

$$
\frac{P+H}{H} \cong \frac{P}{P \cap H}=\frac{P}{P \cap Q}=\frac{P}{T} \cong \frac{A_{2}}{B_{2}} \cong \frac{J}{K}=\frac{J}{H \cap J} \cong \frac{J+H}{H},
$$

so $(P+H) / H$ and $(J+H) / H$ are minimal ideals of $L / H$. It follows that $P+H=J+H$. Similarly, $L / J$ is primitive of type 2 with unique minimal ideal $(H+J) / J$. As $(P+J) / J$ is an ideal of $L / J$ we have $H+J \subseteq P+J \subseteq$ $P+H+J=H+J$, so $P+H=J+H=P+J$.

If $P=J$ then $T=P \cap Q=J \cap H=K$ and $A_{1} / B_{1} \cong_{L} A_{3} / B_{3}$, a contradiction. It follows that $P \neq J$. Let $M, N$ be maximal subalgebras of $L$ such that $K \subseteq M, T \subseteq N, M$ is a common complement of $H / K$ and $J / K$, and $N$ is a common complement of $P / T$ and $Q / T$, so $M_{L}=K$ and $N_{L}=T$. Put $W=P \cap J$ and $X=M \cap N+W$. Then $L=M+H=$ $M+K=N+P=N+Q$ from which it follows that $M \cap W$ is an ideal of $L$ and $M \cap W=K \cap T$. Now $X=L$ implies that $M=M \cap X=$ $M \cap(M \cap N+W)=M \cap N+M \cap W=M \cap N+K \cap T=M \cap N$, a contradiction, as $M \neq N$. Thus $X \neq L$ and

$$
\begin{aligned}
P+X & =P+M \cap N+W=P+M \cap N=P+T+M \cap N \\
& =P+(T+M) \cap N=P+N=L .
\end{aligned}
$$

Similarly, $J+X=L$. 
But $P \cap X$ is an ideal of $X$ and $(P \cap X) / W \subseteq C_{L / W}(J)$, so $P \cap X$ is an ideal of $J+X=L$. Since $P / W \cong(P+J) / J=(H+J) / J$ is a chief factor of $L$ and $W \subseteq P \cap X \subseteq P$, we have $P \cap X=P$ or $P \cap X=W$. The former implies that $P \subseteq X$, which in turn yields that $X=L$, a contradiction. Thus we have $P \cap X=W$. Similarly $J \cap X=W$. Finally, $P / W$ is a minimal ideal of $L / W$ and $C_{L / W}(P / W)=J / W$, and so $L / W$ is primitive of type 3 and $L$-connects $A_{1} / B_{1}$ and $A_{3} / B_{3}$.

We now seek more detailed information about supplemented chief factors.

Proposition 2.5 Let $A / B$ be a chief factor of the Lie algebra $L$ supplemented by the maximal subalgebra $M$, and let $C=C_{L}(A / B)$. Then

(i) $\left(A+M_{L}\right) / M_{L}$ is a minimal ideal of the primitive Lie algebra $L / M_{L}$;

(ii) if $M$ is of type 1 or 3 then each chief factor of $L$ supplemented by $M$ is complemented by $M$;

(iii) if $A / B$ is abelian then $L / M_{L}$ is of type 1 and is isomorphic to the semidirect sum $A / B \rtimes L / C$; and

(iv) if $A / B$ is non-abelian then $L / C$ is primitive of type 2 , $\operatorname{Soc}(L / C) \cong_{L}$ $A / B$, and, if $K$ is a maximal subalgebra supplementing $(A+C) / C$ in $L$, then $K$ is also a supplement to $A / B$ in $L$ and $K_{L}=C$.

\section{Proof.}

(i) It is clear that $L / M_{L}$ is primitive and $B=A \cap M_{L}$. Suppose that $S$ is an ideal of $L$ with $M_{L} \subseteq S \subseteq A+M_{L}$. Then $S=\left(A+M_{L}\right) \cap S=$ $A \cap S+M_{L}$ and $B \subseteq A \cap S \subseteq A$. Then either $A \cap S=B$, in which case $S=M_{L}$, or $A \cap S=A$, in which case $S=A+M_{L}$. Hence $\left(A+M_{L}\right) / M_{L}$ is a minimal ideal of $L / M_{L}$.

(ii) If $M$ is of type 1 or 3 then $\left(A+M_{L}\right) / M_{L}$ is a minimal ideal of $L / M_{L}$, which is primitive of type 1 or 3 and so $M \cap\left(A+M_{L}\right)=M_{L}$. But then $M \cap A=M_{L} \cap A=B$.

(iii) If $A / B$ is abelian then $L / M_{L}$ is primitive of type 1 , in which case $C=A+M_{L}$ and $M / M_{L} \cong L / C$. It follows that $L / M_{L}$ is isomorphic to the semidirect sum $A / B \rtimes L / C$. 
(iv) If $A / B$ is non-abelian then two possibilities arise. If $C=M_{L}$ then $L / M_{L}$ is primitive of type 2 and $\operatorname{Soc}(L / C)=(A+C) / C \cong_{L} A / B$. If $M_{L} \subset C$, then $L / M_{L}$ is primitive of type 3 with minimal ideals $\left(A+M_{L}\right) / M_{L}$ and $C / M_{L}$. In this case $L / C$ is primitive of type 2 and $\operatorname{Soc}(L / C)=(A+C) / C \cong_{L} A / B$.

In either case, let $K$ be a maximal subalgebra supplementing $(A+$ $C) / C$ in $L$. Then $L=A+K$ and $B=A \cap B=A \cap K_{L}$. Hence $K$ is also a supplement of $A / B$ in $L$ and $K_{L}=C$.

In view of the above result, for any chief factor $A / B$ of $L$ we define the primitive algebra associated with $A / B$ in $L$ to be

(i) the semidirect sum $A / B \rtimes\left(L / C_{L}(A / B)\right)$ if $A / B$ is abelian, or

(ii) the factor algebra $L / C_{L}(A / B)$ if $A / B$ is non-abelian.

Let $A / B$ be a supplemented chief factor of $L$ for which $M$ is a maximal subalgebra of $L$ supplementing $A / B$ in $L$ such that $L / M_{L}$ is monolithic and primitive. Note that Proposition 2.5 (iii) and (iv) show that such an $M$ exists; we call $M$ a monolithic maximal subalgebra supplementing $A / B$ in $L$. We say that the chief factor $\operatorname{Soc}\left(L / M_{L}\right)=\left(A+M_{L}\right) / M_{L}$ is the precrown of $L$ associated with $M$ and $A / B$, or simply, a precrown of $L$ associated with $A / B$.

If $A / B$ is a non-abelian chief factor of $L$, then for each maximal subalgebra $M$ of $L$ supplementing $A / B$ in $L$ such that $L / M_{L}$ is a monolithic and primitive, we have that $M_{L}=C_{L}(A / B)$. Therefore the unique precrown of $L$ associated with $A / B$ is

$$
\operatorname{Soc}\left(L / M_{L}\right)=\frac{A+M_{L}}{M_{L}}=\frac{A+C_{L}(A / B)}{C_{L}(A / B)} .
$$

However, if $A / B$ is a complemented abelian chief factor of $L$ and $M$ is a complement of $A / B$ in $L$, then the precrown of $L$ associated with $M$ and $A / B$ is

$$
\operatorname{Soc}\left(L / M_{L}\right)=\frac{A+M_{L}}{M_{L}}=C_{L / M_{L}}\left(\frac{A+M_{L}}{M_{L}}\right)=\frac{C_{L}(A / B)}{M_{L}} .
$$

This raises the question of how many different precrowns are associated with a particular abelian chief factor. For solvable algebras the answer is given by the following result. 
Proposition 2.6 Let $A / B$ be a complemented chief factor of a solvable Lie algebra $L$ over a field $F$ of positive characteristic $p$, and suppose further that $L^{2}$ has nilpotency class less than $p$. Then the map which assigns to each conjugacy class of complements of $A / B$ in $L,\{\exp (a d a)(M): a \in L\}$ say, the common core $M_{L}$ of its elements, induces a bijection between the set of all conjugacy classes of complements of $A / B$ in $L$ and the set of all ideals of $L$ which complement $A / B$ in $C_{L}(A / B)$.

Hence there is a bijection between the set of precrowns of $L$ associated with $A / B$ and the set of all conjugacy classes of complements of $A / B$ in $L$.

Proof. Put $C=C_{L}(A / B)$, and let $M$ be a maximal subalgebra of $L$ such that $L=A+M$ and $A \cap M=B$. Put $N=C \cap M$. Then $N$ is an ideal of $L$ such that $C=A+N$ and $A \cap N=B$. Then $(A+N) / N \cong_{L} A / B$ and $(A+N) / N$ is a self-centralising minimal ideal of $L / N$. By Theorem 1.3, $(A+N) / N$ is complemented in $L / N$ and all complements are conjugate. If $M / N$ is one of these complements, then $N=M_{L}$. Hence the map is surjective.

Let $M$ and $S$ be two complements of $A / B$ in $L$ such that $N=M_{L}=$ $S_{L}$. Then $L / N$ is solvable and primitive such that and $S / N, M / N$ are complements of $\operatorname{Soc}(L / N)=(A+N) / N$. By [8, Theorem 5], there exists an element $a \in A$ such that $\exp (\operatorname{ad} a)(S)=M$. Hence the correspondence is injective.

The maximal subalgebras in a conjugacy class have a common core, by [8, Theorem 4]. Finally observe that, since $A / B$ is abelian, the precrowns of $L$ associated with $A / B$ have a common numerator $C_{L}(A / B)$ and different denominators $M_{L}$, one for each conjugacy class of complements of $A / B$ in $L$.

Proposition 2.7 Let $A_{i} / B_{i}, i=1,2$, be two supplemented chief factors of $L$ that are $L$-connected, and let $C_{i} / R_{i}$ be a precrown associated with $A_{i} / B_{i}$ for $i=1,2$. Then $C_{1}=C_{2}$.

Proof. If $A_{i} / B_{i}$ is abelian, then $C_{1}=C_{L}\left(A_{1} / B_{1}\right)=C_{L}\left(A_{2} / B_{2}\right)=C_{2}$.

If $A_{1} / B_{1}$ and $A_{2} / B_{2}$ are nonabelian but $L$-isomorphic, they have the same precrown, by Proposition 2.5 (iv).

So suppose that there is an ideal $N$ of $L$ such that $L / N$ is primitive of type 3 with minimal ideals $E_{1} / N, E_{2} / N$ such that $E_{1} / N \cong_{L} A_{1} / B_{1}$ and $E_{2} / N \cong{ }_{L} A_{2} / B_{2}$. Then $C_{L}\left(E_{1} / N\right)=E_{2}$ and $C_{L}\left(E_{2} / N\right)=E_{1}$. Hence the precrown associated with $E_{1} / N$ and $A_{1} / B_{1}$ is $\left(E_{1}+E_{2}\right) / E_{2}$, and the precrown associated with $E_{2} / N$ and $A_{2} / B_{2}$ is $\left(E_{1}+E_{2}\right) / E_{1}$. 


\section{Crowns}

Let $A / B$ be a supplemented chief factor of $L$ and put $\mathcal{J}=\left\{M_{L}: M\right.$ is a monolithic maximal subalgebra of $L$ which supplements a chief factor of $L$ which is $L$-connected to $A / B\}$. Let $R=\cap\{N: N \in \mathcal{J}\}$ and $C=$ $A+C_{L}(A / B)$. Then we call $C / R$ the crown of $L$ associated with $A / B$.

Lemma 3.1 Let $\mathcal{J}_{1}=\{N: D / N$ is a precrown associated with a chief factor $L$-connected to $A / B\}, \mathcal{J}_{2}=\left\{M_{L}: M\right.$ is a maximal subalgebra of $L$ supplementing a chief factor $L$-connected to $A / B\}$, and $\mathcal{J}_{3}=\left\{M_{L}: M\right.$ is a maximal subalgebra of $L$ supplementing a chief factor $L$-isomorphic to $A / B\}$. Then

$$
\bigcap\{N: N \in \mathcal{J}\}=\bigcap\left\{N: N \in \mathcal{J}_{1}\right\}=\bigcap\left\{N: N \in \mathcal{J}_{2}\right\}=\bigcap\left\{N: N \in \mathcal{J}_{3}\right\} .
$$

Proof. This follows straightforwardly from Proposition 2.5.

Theorem 3.2 Let $C / R$ be the crown associated with the supplemented chief factor $A / B$ of $L$. Then $C / R=\operatorname{Soc}(L / R)$. Furthermore

(i) every minimal ideal of $L / R$ is a supplemented chief factor of $L$ which is $L$-connected to $A / B$, and

(ii) no supplemented chief factor of $L$ above $C$ or below $R$ is $L$-connected to $A / B$.

In other words, there are $r$ ideals $A_{1}, \ldots, A_{r}$ of $L$ such that

$$
C / R=A_{1} / R \oplus \ldots \oplus A_{r} / R
$$

where $A_{i} / R$ is a supplemented chief factor of $L$ which is $L$-connected to $A / B$ for $i=1, \ldots, r$ and $r$ is the number of supplemented chief factors of $L$ which are $L$-connected to $A / B$ in each chief series for $L$. Moreover, $\phi(L / R)=0$.

Proof. Let $R=N_{1} \cap \ldots \cap N_{r}$ where $C / N_{i}$ are the precrowns associated with chief factors that are $L$-connected to $A / B$ and $r$ is minimal with respect to this property. Then

$$
\theta: \frac{C}{R}=\frac{C}{N_{1} \cap \ldots \cap N_{r}} \rightarrow \frac{C}{N_{1}} \oplus \ldots \oplus \frac{C}{N_{r}}
$$

given by $\theta\left(c+\left(N_{1} \cap \ldots \cap N_{r}\right)\right)=\left(c+N_{1}, \ldots, c+N_{r}\right)$ is an $L$-monomorphism. Moreover, $C=N_{i}+\left(N_{1} \cap \ldots \cap N_{i-1}\right)$ for $i \leq r$, from the minimality of $r$, and so

$$
\frac{N_{1} \cap \ldots \cap N_{i-1}}{N_{1} \cap \ldots \cap N_{i}} \cong_{L} \frac{C}{N_{i}} .
$$


It follows that the chain

$$
R=N_{1} \cap \ldots \cap N_{r} \subseteq N_{1} \cap \ldots \cap N_{r-1} \subseteq \ldots \subseteq N_{1} \subseteq C
$$

is part of a chief series for $L$ in which each chief factor is $L$-connected to $A / B$. Hence $\operatorname{dim}(C / R)=r \operatorname{dim}(A / B)$ and $\theta$ is an isomorphism.

Suppose that $E / F$ is a supplemented chief factor of $L$ which is $L$ connected to $A / B$ and let $M$ be a maximal subalgebra of $L$ that is a supplement of $E / F$ in $L$. Then $E \subseteq C$, by Proposition 2.7. However, $E \nsubseteq R$, since $R \subseteq M_{L}$. It follows that no supplemented chief factor of $L$ over $C$ or below $R$ is $L$-connected to $A / B$.

By Theorem 2.3 the number of supplemented chief factors $L$-connected to $A / B$ in each chief series of $L$ is an invariant of $L$ and coincides with the length of any section of chief series between $R$ and $C$.

Next suppose that $D / R$ is a minimal ideal of $L / R$ and that $D \nsubseteq C$. Then $D \cap C=R$ and $D \subseteq C_{L}\left(A_{1} / R\right)$. But $A_{1} / R$ is $L$-connected to $A / B$, so $D \subseteq C_{L}\left(A_{1} / R\right) \subseteq C$, by Proposition 2.7.

Finally, $\phi(L / R)=0$ since every minimal ideal of $L / R$ is supplemented.

Corollary 3.3 Two supplemented chief factors of $L$ define the same crown if and only if they are L-connected.

Proof. This is clear, since the crown associated with a supplemented chief factor is a direct sum of supplemented components.

Proposition 3.4 For any Lie algebra $L$ we have

$\cap\{S$ : there is a non-abelian crown $R / S$ of $L\}$

$=\cap\left\{M_{L}: M\right.$ is maximal in $L$ and $L / M_{L}$ is primitive of type 2$\}$

$=\cap\left\{M_{L}: M\right.$ is maximal in $L$ and $L / M_{L}$ is primitive of type 2 or 3$\}$

$=\cap\left\{C: C=C_{L}(A / B), A / B\right.$ a non-abelian chief factor of $\left.L\right\}$

$=\Gamma$, the solvable radical of $L$.

Proof. It follows from Lemma 3.1 and Theorem 3.2 that the given intersections all yield the same ideal, $J$ say. Let $A / B$ be a chief factor of $L$ below $J$. If $A / B$ is non-abelian we have $A \subseteq J \subseteq C_{L}(A / B)$, a contradiction, so $J \subseteq \Gamma$. Moreover, if $R / S$ is a non-abelian crown of $L$, then $(S+\Gamma) / S$ is a solvable ideal of $L / S$ and so is trivial, since $R / S=\operatorname{Soc}(L / S)$, by Theorem 3.2. It follows that $\Gamma \subseteq S$, whence $\Gamma \subseteq J$. 
Theorem 3.5 Let $L$ be a solvable Lie algebra, and let $C / R=\bar{C}$ be the crown associated with a supplemented chief factor of $L$. Then $\bar{C}$ is complemented in $\bar{L}$, and any two complements are conjugate by an automorphism of the form $1+$ ad a for some $a \in \bar{C}$.

Proof. For simplicity we will assume that $R$ has been factored out and write the crown simply as $C$. Then $C=$ Asoc $L$ and $\phi(L)=0$, so $L=C \dot{+} U$ for some subalgebra $U$ of $L$, by [5, Theorem 7.3].

Let $\operatorname{Asoc} L=A_{1} \oplus \ldots \oplus A_{n}$ in $L$, where $A_{i}$ is a minimal ideal of $L$ for $i=1, \ldots, n$. Then $\operatorname{Asoc} L=N$, where $N$ is the nilradical of $L$, by [5, Theorem 7.4], and $C_{L}(N)=N$. Now $A_{i} \cong_{L} A_{j}$ for each $1 \leq i, j \leq n$, and so $C_{L}\left(A_{i}\right)=N$ for $i=1, \ldots, n$. Let $D / N$ be a minimal ideal of $L / N$. Then there exists $d \in D$ which does not act nilpotently on $N$. Let $L=E_{L}(d) \dot{+} L_{1}$ be the Fitting decomposition of $L$ relative to ad $d$. Clearly $L_{1} \subseteq N$, and $L_{1}$ is an ideal of $L$. Without loss of generality we can assume that $L_{1}=A_{1} \oplus \ldots \oplus A_{r}$, where $r \leq n$. Since $\left[L_{1}, d\right]=L_{1}$ it follows that $\left[A_{i}, d\right]=A_{i}$ for each $i=1, \ldots, r$, whence $[N, d]=N$, since the $A_{i}^{\prime} s$ are $L$ isomorphic to each other. Thus $L_{1}=N$ and we can assume that $U=E_{L}(d)$.

Let $V$ be another complement of $C$ in $L$. Then there exists $v \in V$ such that $v=d+n$ for some $n \in N$. Now $N=[N, d]$, so $n=[a, d]$ for some $a \in N$. Thus $v=d+[a, d]=d(1+\operatorname{ad} a)$. But $1+\operatorname{ad} a$ is an automorphism of $L$, and so

$$
U(1+\operatorname{ad} a)=E_{L}(d)(1+\operatorname{ad} a)=E_{L}(v) \supseteq V,
$$

since $v$ corresponds to $d$ in the isomorphism $V \rightarrow L / N \rightarrow U$. But $E_{L}(v)$ and $V$ are both complements to $N$ in $L$, and so $E_{L}(v)=V$, and the result follows.

Let

$$
0=L_{0} \subset L_{1} \subset \ldots \subset L_{n}=L
$$

be a chief series for $L$. We define the set $\mathcal{I}$ by $i \in \mathcal{I}$ if and only if $L_{i} / L_{i-1}$ is not a Frattini chief factor of $L$. For each $i \in \mathcal{I}$ put

$$
\mathcal{M}_{i}=\left\{M \text { is a maximal subalgebra of } L: L_{i-1} \subseteq M \text { but } L_{i} \not \subset M\right\} .
$$

Then $B$ is a prefrattini subalgebra of $L$ if

$$
B=\bigcap_{i \in \mathcal{I}} M_{i} \text { for some } M_{i} \in \mathcal{M}_{i}
$$

It was shown in [7] that the definition of prefrattini subalgebras does not depend on the choice of chief series. 
Theorem 3.6 Let $L$ be a solvable Lie algebra. Then the prefrattini subalgebras of $L$ are precisely the intersections of the complements of the crowns, one complement being taken from each crown.

Proof. Let $L_{i} / L_{i-1}$ be a non-Frattini, and hence supplemented, chief factor of $L$, and let its crown $C / R=A_{1} / R \oplus \ldots \oplus A_{r} / R$ be complemented by $K / R$. Then $M_{i}=A_{1} \oplus \ldots \oplus \hat{A}_{i} \oplus \ldots \oplus A_{r} \dot{+} K$ (where the 'hat' is over a term that is omitted from the sum) is a maximal subalgebra of $L$ such that $R \subseteq M_{i}$ but $A_{i} \not \subseteq M_{i}$. It is clear that if we intersect all such subalgebras over each of the crowns then we get a prefrattini subalgebra of $L$, and that this intersection is equal to the intersection of the complements $K$, one for each crown.

Moreover, if $M$ is a maximal subalgebra with $R \subseteq M$ but $A_{i} \nsubseteq \nsubseteq M$, then $L=A_{i} \dot{+} M$ and $M \cong L / A_{i} \cong A_{1} \oplus \ldots \oplus \hat{A}_{i} \oplus \ldots \oplus A_{r} \dot{+} K$. It follows that we get all prefrattini subalgebras this way.

Theorem 3.7 Let $L$ be a solvable Lie algebra, $A / B$ a supplemented chief factor of $L, C / R$ the crown associated with $A / B$ and $K / R$ a complement for $C / R$. Then $K$ avoids every chief factor that is $L$-connected to $A / B$ and covers the rest.

Proof. We have $L=C+K, C \cap K=R$ and $C^{2} \subseteq R \subset K$. Let $E / F$ be a supplemented chief factor of $L$. Then $F+[C, E]=F$ or $E$. Suppose first that $F+[C, E]=F$. Then $[C, E] \subseteq F$. Note that this case must occur if $E / F$ is $L$-connected to $A / B$, since then $C=C_{L}(A / B)=C_{L}(E / F)$. But now $F+K \cap E$ is an ideal of $L$ and so $F+K \cap E=F$ or $E$. The former implies that $K \cap E \subseteq K \cap F$, whence $K \cap E=K \cap F$ and $K$ avoids $E / F$.

The latter yields that $F+K=E+K$ and $K$ covers $E / F$. In this case, if $E / F$ is $L$-connected to $A / B$ we have $R \subseteq F$ and $E=F+K \cap E$. But $K \cap E \subseteq K \cap C=R$, from which $E \subseteq F+R=F$, a contradiction. So this case only occurs when $E / F$ is not $L$-connected to $A / B$.

The remaining possibility (which also only occurs when $E / F$ is not $L$ connected to $A / B)$ is that $F+[C, E]=E$. Then $[C, E] \subseteq F+[C,[C, E]] \subseteq$ $F+C^{2} \subseteq F+R \subseteq F+K$, giving $E \subseteq F+K$. Thus $E+K=F+K$ and $K$ covers $E / F$.

\section{References}

[1] R.E. Block, 'Differentiably simple algebras', Bull. Amer. Math. Soc. 74 (1968), 1086 - 1090. 
[2] A. Ballester-Bolinches and L.M. Ezquerro, 'Classes of Finite Groups', Mathematics and its Applications 584 (2006), Springer.

[3] H. Strade and R. Farnsteiner, 'Modular Lie algebras and their Representations', Marcel-Dekker, New York and Basel 1988.

[4] J. Lafuente, 'Nonabelian crowns and Schunck classes of finite groups', Arch. Math. 42 (1984), 32-39.

[5] D.A. Towers, 'A Frattini theory for algebras', Proc. London Math. Soc. (3) 27 (1973), 440-462.

[6] D.A. Towers, 'On Lie algebras in which modular pairs of subalgebras are permutable', J. Algebra 68 (1981), 369-377.

[7] D.A. Towers, 'Complements of intervals and prefrattini subalgebras of solvable Lie algebras', Proc. Amer. Math. Soc. 141 (2013), 1893-1901.

[8] D.A. Towers, 'On conjugacy of maximal subalgebras of solvable Lie algebras', Comm. Alg. 42 (3) (2014), 1350-1353. 